\title{
Extensive latrogenic Aortic Dissection During Renal Angioplasty: Successful Treatment with a Covered Stent-Graft
}

\author{
M. Rasmus, ${ }^{1}$ R. Huegli, ${ }^{1}$ A.L. Jacob, ${ }^{1}$ M. Aschwanden, ${ }^{2}$ D. Bilecen ${ }^{1}$ \\ ${ }^{1}$ Interventional Radiology, University Hospital of Basel, Petersgraben 4, 4056 Basel, Switzerland \\ ${ }^{2}$ Department of Angiology, University Hospital of Basel, Basel, Switzerland
}

\begin{abstract}
An extensive iatrogenic aortic type B dissection during percutaneous transluminal renal angioplasty (PTRA) for bilateral renal artery stenosis was treated with a covered stent placed in the right renal artery. Control angiography confirmed closure of the entry. Postprocedural CT demonstrated a thick intramural hematoma (IMH) up to the left subclavian artery. CT follow-up at 8 months showed an almost complete resorption of the IMH. While medical treatment is the standard therapy for type B dissections, closure of the intimal tear with a covered stent may be an additional option in extensive cases during PTRA.
\end{abstract}

Key words: Angioplasty-Aortic type B dissection-Complications-Covered stent-Renal artery stenosis

Type B aortic dissection as a possible severe complication of percutaneous renal artery angioplasty (PTRA) has been observed several times [1-4]. Standard treatment consists of antihypertensive drug therapy. We report a case in which an iatrogenic intimal tear at the level of the origin of the right renal artery was sealed with a covered stent-graft in the same procedure. Short and midterm follow-up with CT showed subtotal exclusion of the dissection and nearly complete resorption of the intramural hematoma.

\section{Case Report}

A 58-year-old man with hypertension and bilateral stenosis of both renal arteries, diagnosed with Doppler ultrasound, presented for elective PTRA. He had been pretreated with atenolol $(2 \times 50 \mathrm{mg} /$ day $)$, simvastatin $(40 \mathrm{mg} /$ day), and acetylsalicylic acid (100 mg/day). Serum creatinine was normal $(100 \mu \mathrm{mol} / \mathrm{l})$.

Aortic flush angiography showed bilateral renal artery stenoses close to the respective origins but not of the ostial type (Fig. 1A). Bilateral PTRA was therefore planned. First, the higher grade stenosis of the left renal artery was treated. After PTRA with a $6 \mathrm{~mm} \times 20 \mathrm{~mm}$ balloon (Fox, Abbott Vascular Devices, Redwood City, CA, USA), the simultaneously measured intra-arterial systolic pressure gradient decreased from $25 \mathrm{mmHg}$ to 2 $\mathrm{mmHg}$. The morphologic appearance was also satisfactory.

The systolic gradient of the right renal artery was $20 \mathrm{mmHg}$ after selective catheterization. PTRA was again performed with the same 6

Correspondence to: PD Dr. D. Bilecen; email: dbilecen@uhbs.ch $\mathrm{mm} \times 20 \mathrm{~mm}$ balloon. The postinterventional contrast examination showed elastic recoil of the stenosis and a large dissection of the aortic wall extending from the renal artery in cephalad and distal directions (Fig. 1B). The systolic gradient increased to $25 \mathrm{mmHg}$. Primarily to treat the stenosis, a $6 \mathrm{~mm} \times 17 \mathrm{~mm}$ renal stent (Renal Jostent, Abbott Vascular Devices) was implanted. Control angiography showed a correction of the stenosis and a somewhat decreased but still active inflow of contrast agent into the false lumen (Fig. 1C). Therefore an additional covered stent (6 $\mathrm{mm} / 28 \mathrm{~mm}$ Jograft, Abbott Vascular Devices) was placed through the first stent into the proximal renal artery to seal off the entry completely. The systolic pressure gradient across the renal artery stenosis decreased to zero, and angiography showed no further inflow into the false lumen (Fig. 1D).

After initial PTRA the patient started to complain of increasing thoracic pain and was treated with analgosedation. He was then brought to the intensive care unit for further observation and strict blood pressure control with a target systolic pressure of $120 \mathrm{mmHg}$.

A CT examination was performed to assess the extent of the dissection about $2 \mathrm{hr}$ after PTRA. A retrograde type B dissection up to the origin of the left subclavian artery with almost complete thrombosis and no active flow into the thoracic part was diagnosed (Figs. 2A, 3A). In the anterograde direction the dissection reached to about $2 \mathrm{~cm}$ above the iliac bifurcation and was partially perfused. The stent protruded through the false lumen into the aortic lumen. Two days later a second CT control was done (Fig. 2B, 3B) because a blood pressure gradient between the right and the left arm was noted for the first time and further retrograde dissection was suspected. CT at this time demonstrated a stable, predominantly thrombosed dissection and a previously unnoticed moderate stenosis of the right subclavian artery as the cause of the pressure gradient.

In the days after the intervention the patient suffered from minor respiratory problems due to basal dystelectasis. The further clinical course was uneventful, but prolonged due to a temporary increase in both Creactive-protein and transaminases, without further clinical signs of infection or liver insufficiency. The patient was discharged on the 11th postinterventional day. C-reactive protein and liver parameters normalized shortly afterward.

At the 2 months follow-up CT showed subtotal shrinkage of the thrombosed thoracic false lumen (Fig. 2C, 3C). From the celiac axis down to about $2 \mathrm{~cm}$ above the bifurcation a residual thrombosed dissection was seen. A small residual perfused volume was visible in the abdominal aorta. The last CT evaluation after 8 months showed a nearly complete resorption of the type B dissection (Fig. 2D, 3D), paralleled by a slight protrusion of the covered stent into the aortic lumen. Neither signs of aneurysmatic expansion (Table 1) nor an increase in the creatinine level above the normal range appeared at any time. Arterial hypertension was satisfactorily controlled with adapted blood pressure medication. 

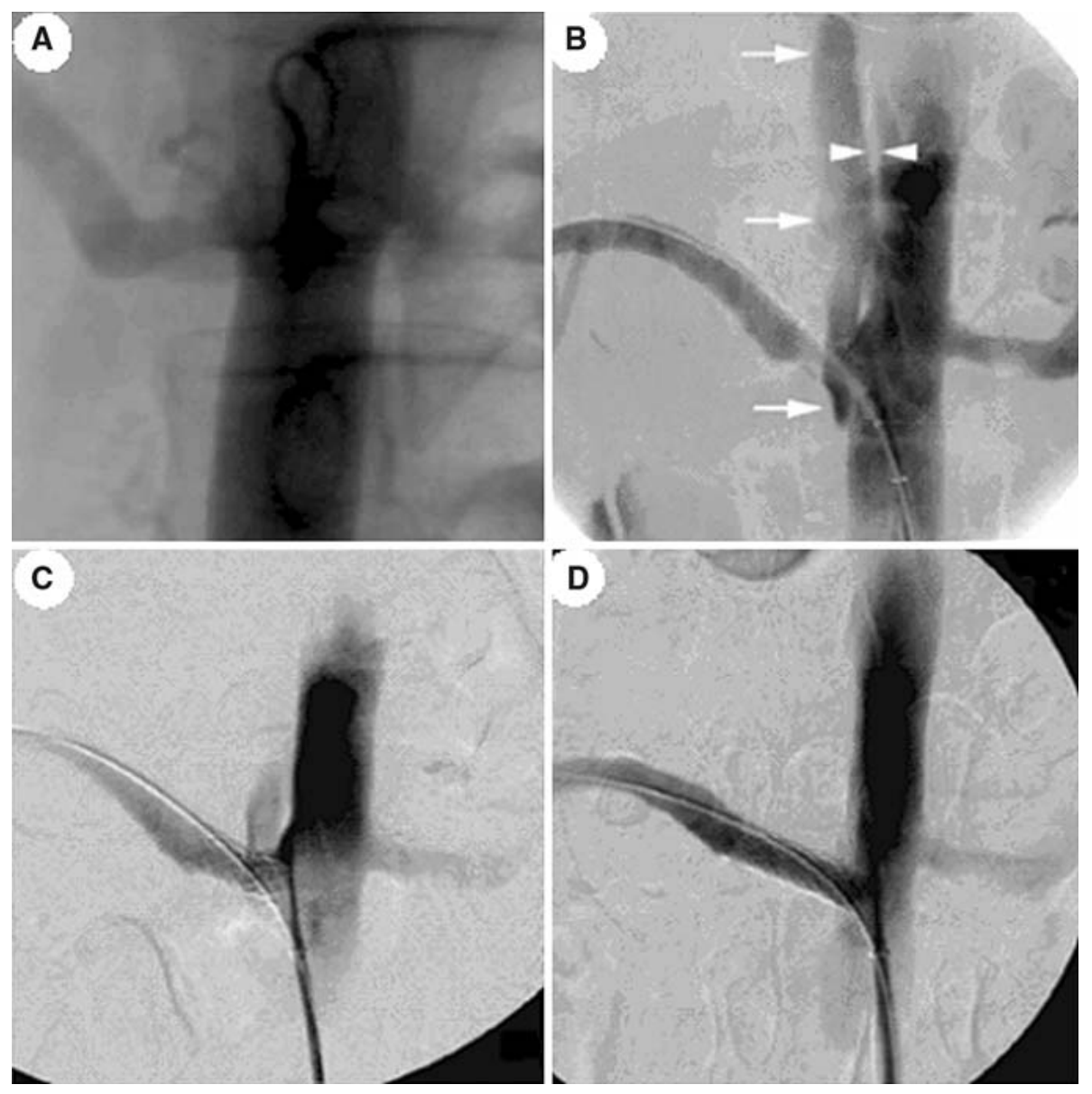

Fig. 1. Aortography and digital subtraction aortograms: A high-grade stenosis of the renal artery on the right side close to the origin but not of the ostial type; B extensive dissection (arrows) after PTRA on the right. An intimal flap (arrowheads) separates the true and false lumens; C decreased contrast agent inflow into the false lumen after positioning of a first uncovered stent; $\mathbf{D}$ complete sealing after positioning of the second covered stent.
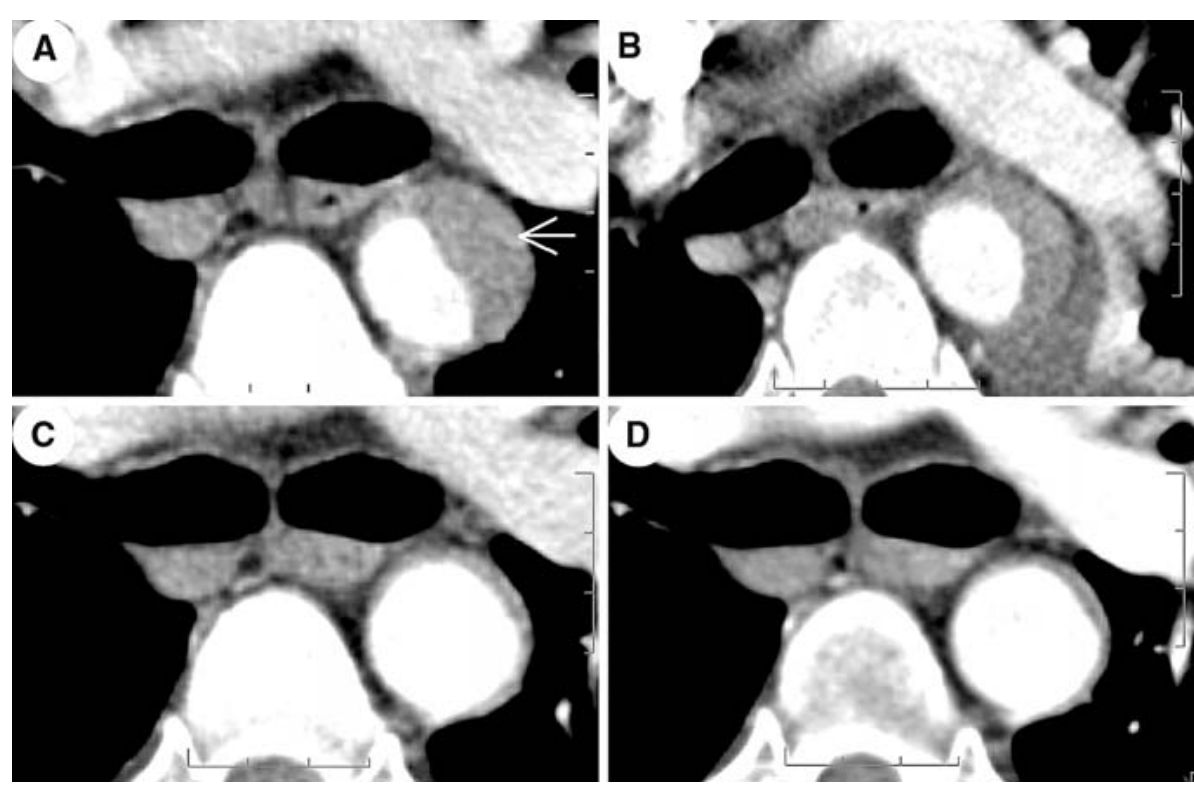

Fig. 2. Serial axial slices at the height of the tracheal bifurcation, A $2 \mathrm{hr}$, B 2 days, $\mathbf{C}$ 2 months, and $\mathbf{D} 8$ months after intervention, showing the dissectional thrombosis (arrow).

\section{Discussion}

Aortic dissection during PTRA is a rare but serious complication [14]. Immediate treatment with closure of the entry with a covered stent within the renal artery has not previously been reported.
In our case the use of a second covered stent after a first stenting with an uncovered stent resulted in a nearly complete shrinkage of the false lumen within 8 months. These findings may be interpreted as the consequence of the closure of the entry in 

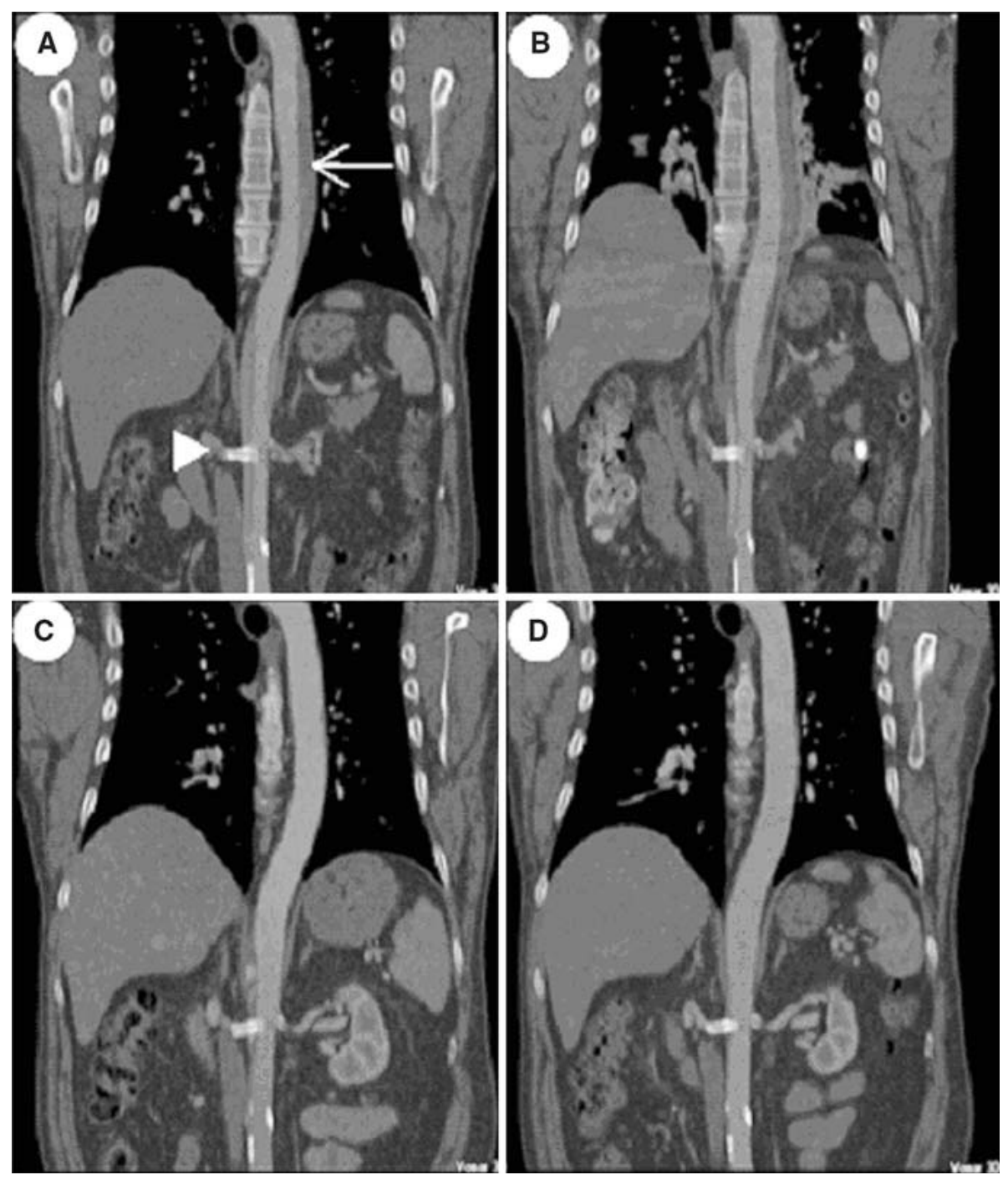

Fig. 3. CT curved reconstruction of the abdominal aorta descendens, A $2 \mathrm{hr}, \mathbf{B}$ 2 days, $\mathbf{C} 2$ months, and $\mathbf{D} 8$ months after dissection, showing the nearly complete regression of the dissection (arrow). The stent can be seen within the right renal artery (arrowhead).

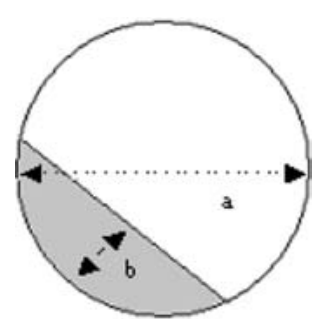

Table 1. Measurement of aortic diameter $(a)$ and width of the dissection (b) in millimeters at the height of the tracheal bifurcation (TB), outlet of the artery mesenterica superior (MS), and three slices distal to the right renal artery (DAR) measured $2 \mathrm{hr}(0 \mathrm{~d}), 2$ days (2d), 2 months ( $2 \mathrm{~m})$, and 8 months (8m) after dissection

\begin{tabular}{rlrrrr}
\hline & & Od & $2 \mathrm{~d}$ & $2 \mathrm{~m}$ & $8 \mathrm{~m}$ \\
\hline$a$ & TB & 29 & 30 & 27 & 27 \\
& MS & 24 & 28 & 28 & 24 \\
$b$ & DAR & 21 & 22 & 22 & 21 \\
& TB & 15 & 11 & 4 & 3 \\
& MS & 12 & 12 & 11 & 4 \\
& DAR & 10 & 10 & 7 & 3 \\
\hline
\end{tabular}

combination with continuing medical treatment, as spontaneous remission is not expected.

Initial diagnosis of bilateral renal artery stenosis in this patient was made with Doppler ultrasound-recently recommended as the most cost-effective screening tool for renal artery stenosis-followed by magnetic resonance angiography and computed tomographic angiography [12].

The number of aortic dissections after renal angioplasty is too small to name potential etiologic factors. In a retrospective comparison between spontaneous aortic dissections and iatrogenic aortic dissections (including diagnostic angiography, interventions, and surgery) patients with iatrogenic dissection were older and tended to have a higher incidence of atherosclerosis [10].

In cases of spontaneous aortic type B dissections, severe associated complications such as death, rupture, visceral ischemia, lower limb ischemia, and others have been described [5]. Even though these complications might occur in those rare cases of 
iatrogenic dissection as well, immediate recognition may provide the advantage of much earlier treatment. Thus mid- and long-term outcomes of iatrogenic dissections may be hypothesized to be better than those after spontaneous dissections.

Thrombosis and shrinkage of a false lumen after closure of the entry is a known phenomenon [6-8], even though it has not previously been reported after iatrogenic dissection.

In spontaneous type $\mathrm{B}$ aortic dissections the absence of a patent false lumen and an aortic diameter of less than $40 \mathrm{~mm}$ [5] are good prognostic parameters. Accordingly we hope that the closure of the entry with subsequent thrombosis will ameliorate the patient's long-term prognosis.

Generally, conservative medical treatment in patients with type $\mathrm{B}$ dissection is preferred if no complications such as aneurysm formation and rupture, visceral ischemia or limb ischemia occur [5]. Accordingly in 3 of 4 published cases of iatrogenic type B dissection during PTRA conservative treatment was performed [1, $2,4]$, with only one patient undergoing surgical treatment [3].

A similar procedure to that in our case, with immediate entry closure by positioning of a second stent in the renal artery, was reported by Bloch et al. [2] and more recently by Warakaulle et al. [11]. In contrast to our case, uncovered stents were used also for the second stenting. In the case reported by Bloch et al., despite primary angiographically confirmed sealing of the dissection, clinical signs of an exacerbation appeared. Control CT revealed an enlarged dissection approximately $90 \mathrm{~min}$ after intervention. The authors hypothesized that the positioning of a second stent might have caused the (re-)dissection because of repeated vessel expansion. In the other case a second uncovered stent was positioned more proximally with protrusion into the aortic lumen of $2-3 \mathrm{~mm}$, which led to thrombosis of the false lumen at control CT after 1 day, even though the dissection flap had still been evident during the angiographic control. The mid-term outcome was not reported.

In our case tear closure was probably immediately successful because of the use of a covered stent which provided a tight seal.

The protrusion of the covered stent into the aortic lumen after shrinkage of the false lumen without further complication such as restenosis, thrombosis or stent dislocation should be mentioned. Other cases of renal artery stent protrusion have been reported [2,
9, 11]. As in our case no further complication occurred due to protrusion in the mid-term follow-up. To the best of our knowledge no complications due to moderate stent protrusion have been reported.

We conclude that closure of an iatrogenic tear with a covered stent was successful in this case of an iatrogenic aortic dissection during PTA and may represent an additional therapeutic option in these rare cases.

\section{References}

1. Haesemeyer SW, Vedantham S, Braverman A (2005) Renal artery stent placement complicated by development of a Type B aortic dissection. Cardiovasc Intervent Radiol 28:98-101

2. Bloch MJ, Trost DW, Sos TA (2001) Type B aortic dissection complicating renal artery angioplasty and stent placement. J Vasc Interv Radiol 12:517-520

3. Dorsey DM, Rose SC (1993) Extensive aortic and renal artery dissection following percutaneous transluminal angioplasty. J Vasc Interv Radiol 4:493-495

4. Gendler R, Mitty HA (1993) Case report: evolution of a type B aortic dissection following renal artery angioplasty. Mt Sinai J Med 60:330332

5. Onitsuka S, Akashi H, Tayama K, et al. (2004) Long-term outcome and prognostic predictors of medically treated acute type B aortic dissections. Ann Thorac Surg 78:1268-1273

6. Nienaber CA, Fattori R, Lund G, et al. (1999) Nonsurgical reconstruction of thoracic aortic dissection by stent-graft placement. $\mathrm{N}$ Engl J Med 340:1539-1545

7. Kato N, Shimono T, Hirano T, et al. (2003) Stent-graft detachment from aortic wall after stent-graft repair of acute aortic dissection. Vasc Surg 38:1130-1131

8. Dake MD, Kato N, Mitchell RS, et al. (1999) Endovascular stent-graft placement for the treatment of acute aortic dissection. N Engl J Med 340:1546-1552

9. Beek FJ, Kaatee R, Beutler JJ, et al. (1997) Complications during renal artery stent placement for atherosclerotic ostial stenosis. Cardiovasc Intervent Radiol 20:184-190

10. Januzzi JL, Sabatine MS, Eagle KA, et al. (2002) Iatrogenic aortic dissection. Am J Cardiol 89:623-626

11. Warakaulle DR, Uberoi R (2006) Renal artery stent placement complicated by the development of an aortic dissection. Cardiovasc Intervent Radiol 29:315-316

12. White CJ (2006) Catheter-based therapy for atherosclerotic renal artery stenosis. Circulation 113:1464-1473 